\title{
Developing our Next Generation BioPhotonics Workstation
}

\author{
Bañas, Andrew Rafael; Palima, Darwin; Tauro, Sandeep; Glückstad, Jesper
}

Published in:

Proceedings of SPIE - The International Society for Optical Engineering

Link to article, DOI:

$10.1117 / 12.877157$

Publication date:

2011

Document Version

Publisher's PDF, also known as Version of record

Link back to DTU Orbit

Citation (APA):

Bañas, A. R., Palima, D., Tauro, S., \& Glückstad, J. (2011). Developing our Next Generation BioPhotonics Workstation. Proceedings of SPIE - The International Society for Optical Engineering, 7950, 795000.

https://doi.org/10.1117/12.877157

\section{General rights}

Copyright and moral rights for the publications made accessible in the public portal are retained by the authors and/or other copyright owners and it is a condition of accessing publications that users recognise and abide by the legal requirements associated with these rights.

- Users may download and print one copy of any publication from the public portal for the purpose of private study or research.

- You may not further distribute the material or use it for any profit-making activity or commercial gain

- You may freely distribute the URL identifying the publication in the public portal

If you believe that this document breaches copyright please contact us providing details, and we will remove access to the work immediately and investigate your claim 


\title{
Developing our Next Generation BioPhotonics Workstation
}

\author{
A. Bañas, D. Palima, S. Tauro and J. Glückstad \\ DTU Fotonik, Dept. of Photonics Engineering, \\ Programmable Phase Optics \\ DK-2800 Kgs. Lyngby, Denmark \\ Email: jesper.gluckstad@,fotonik.dtu.dk \\ URL: www.ppo.dk
}

\begin{abstract}
Optical trapping and manipulation have established a track record for cell handling in small volumes. However, this cell handling capability is often not simultaneously utilized in experiments using other methods for measuring single cell properties such as fluorescent labeling. Such methods often limit the trapping range because of high numerical aperture and imaging requirements. To circumvent these issues, we are developing a BioPhotonics Workstation platform that supports extension modules through a long working distance geometry. Furthermore, a long range axial manipulation range is achieved by the use of counter-propagating beam traps coupled with the long working distance. This geometry provides three dimensional and real time manipulation of a plurality of traps - currently 100 independently reconfigurable - facilitating precise control and a rapid response in all sorts of optical manipulation undertakings. We present ongoing research activities for constructing a compact next generation BioPhotonics Workstation.
\end{abstract}

Keywords: Spatial light modulator, optical manipulation, optical correlation, Generalized Phase Contrast

\section{INTRODUCTION}

Since Ashkin's pioneering demonstration of optical trapping [1], the ability to use light for moving microscopic structures have been applied to many studies, especially in biological applications [2]. When a refractive or reflective object changes the direction of incident light, light's momentum is changed, resulting in a force that moves the object if it small enough. Due to its relative simplicity, most of the recent optical manipulation schemes are based on the principle of trapping particles inside a strongly focused continuous wave laser beam and movement was done simply by translating the laser focus - a technique now commonly termed optical tweezers (Fig 1.a). Such traps require light intensity gradients, and typically take advantage high numerical aperture focusing. Recently computer addressable spatial light modulators became an indispensible tool for optical manipulation. Incident light can be divided into arbitrary shapes and locations, making it easy to have multiple traps on the same setup. The convenience of programming an SLM through a computer with real time input from a user interface or pre-calculated trap trajectories also enables dynamic optical trapping. This allows a plurality of reconfigurable traps capable of translating simple bead like structures, and rotating structures with multiple handles about the optical axis. SLM controlled optical tweezers perform well for manipulation at the focal plane, however, the use of high NA focusing limits the axial range of motion as high intensity light is strongly localized.

Complex Light and Optical Forces V, edited by David L. Andrews, Enrique J. Galvez, Jesper Glückstad, Proc. of SPIE Vol. 7950, 79500O - @ 2011 SPIE · CCC code: 0277-786X/11/\$18 · doi: 10.1117/12.877157 


\section{MANIPULATION WITH COUNTER-PROPAGATING BEAM TRAPS}

We extend optical trapping to the axial direction by using a low NA counter-propagating beam geometry on our BioPhotonics Workstation. Counter-propagating (CP) traps take advantage of the long working distance wherein the light intensity does not deviate as much as compared to the high NA focusing. Trapped particles are translated axially by varying the relative intensities of the counter propagating beams (Fig 1.b). This axial degree of freedom enables more interactive manipulation flipping of planar microstructures [3] and lifting puzzle pieces of reconfigurable microenvironments [4]. Therefore, with the concerted transverse and axial manipulation provided by gradient forces and scattering forces respectively $3 \mathrm{D}$ optical manipulation is achieved while overcoming the short range limitations of high NA optical tweezer based trapping [5].

Long range 3D optical manipulation enables a host of novel applications, especially in microscopic biological studies. For example, microscopic scaffoldings fabricated with two-photon polymerization (2PP) can be reassembled with multiple traps, simulating biological microenvironments that can influence the behavior of cells [4] (Fig 2). With micro or nano-fabrication processes such as $2 \mathrm{PP}$ or chemical micro-assembly, specially designed microscopic tools can be driven around biological samples for probing or sending stimulus. Similar to freely movable hand tools, 3D controllable micro-tools can be used to trigger biological, chemical or mechanical reactions in a localized and controlled manner.
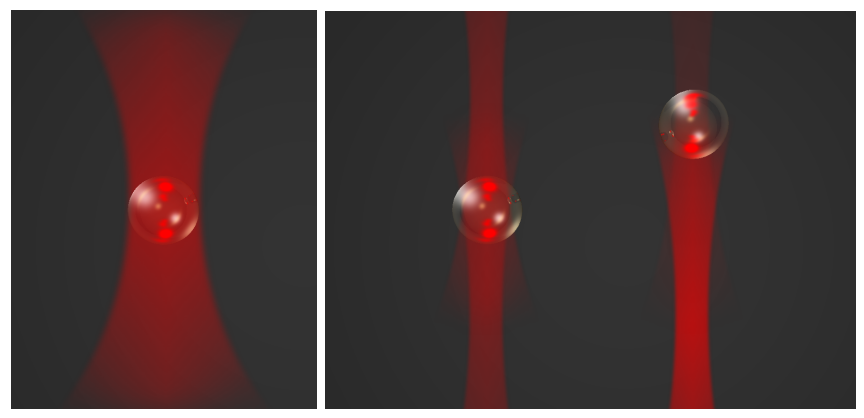

Figure 1. (a) Conventional optical tweezers. (b) Counter-propagating beam traps showing how axial translation is achieved by changing the relative intensities of the opposing beams in each trap.
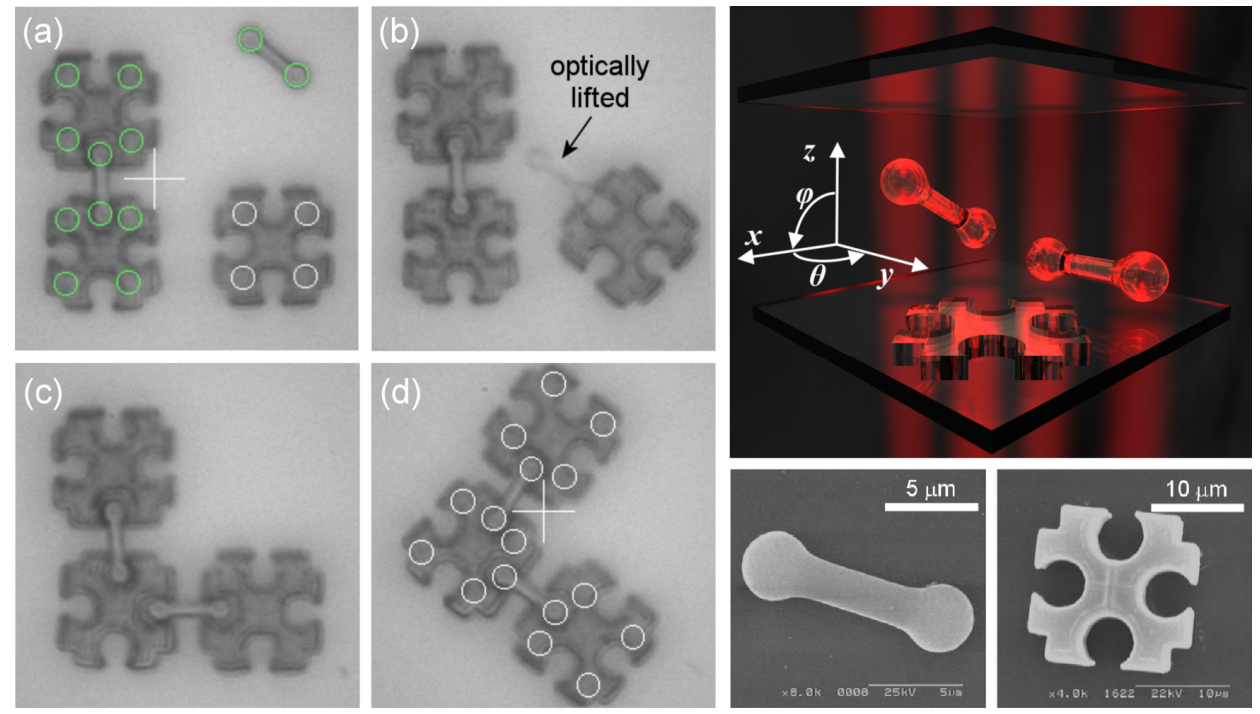
Fig. 2. Snapshots from an acquired video illustrating optical microassembly of reconfigurable microenvironments fabricated using two-photon photo-polymerized components. Images are from Ref. 4 and were featured by Nature Photonics in June 2009. (c) Combined with microstructures with multiple handles, counter-propagating traps enables manipulation with 6 degrees of freedom.

\section{THE BIOPHOTONICS WORKSTATION}

The schematics of the BioPhotonics Workstation is shown in Fig. 3 and its configuration and optical modules has been dealt with extensively so we outline only the pertinent features here. Two independently addressable regions of a spatial beam modulator are optically mapped and relayed as a plurality of reconfigurable counter-propagating beams in the sample. The scaling between the spatially light modulating pixels and the sample plane are specified by choosing appropriate focal lengths of the relaying lenses. The user can independently control the number, size, shape, intensity and spatial position of each CP-beamlet through a LabView interface. Each CP-beamlet can independently trap and manipulate a microscopic object, in parallel with the other trapping CP-beamlets.

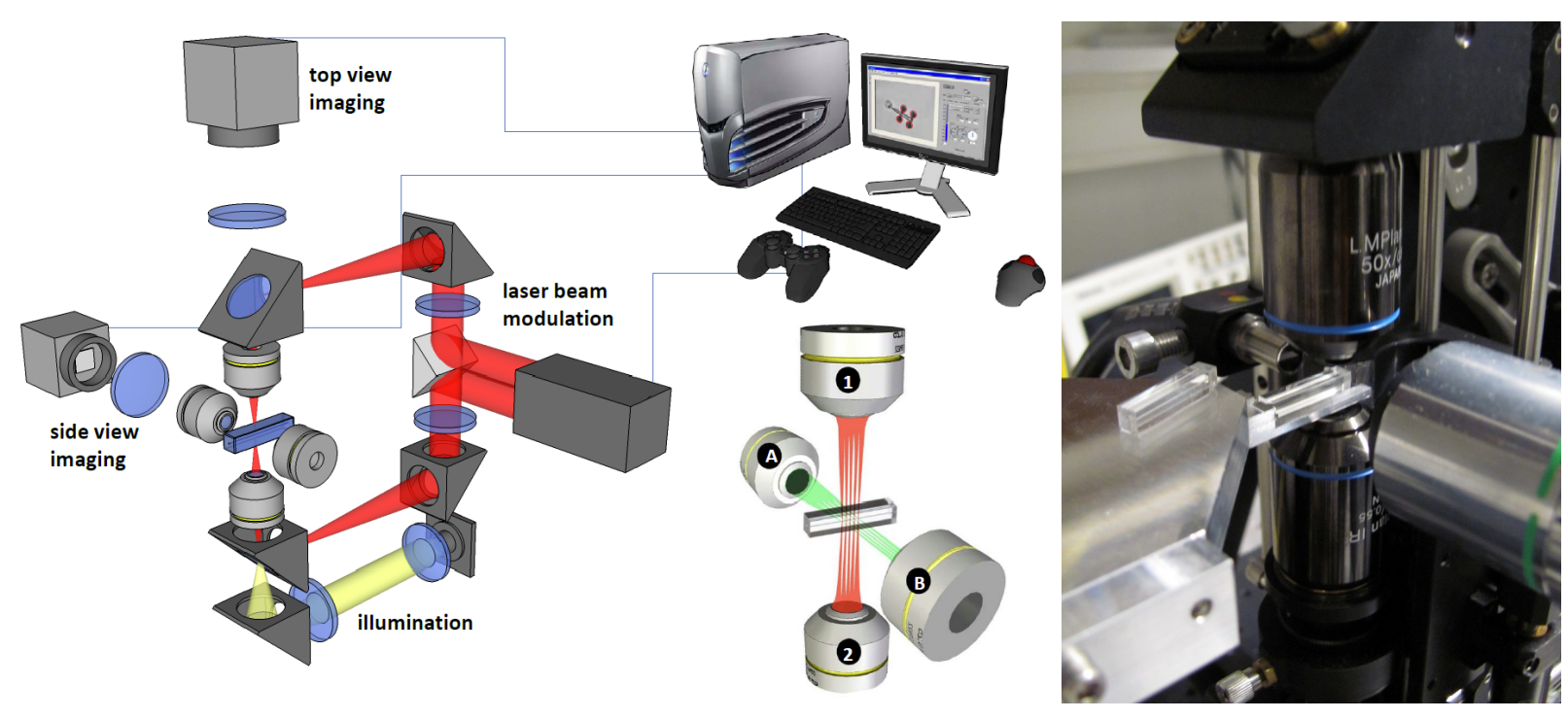

Figure 3. Schematics of the BioPhotonics workstation. The long working distance allows an extra microscope to be mounted perpendicular for side view or for an independent optical setup. The laser source is modulated and shaped by a spatial light modulator; the upper and lower parts of the beam are separated and projected into the sample from opposite directions. A periscopic design, with two mirrors in each arm, simplifies the optical alignment.

Computational overhead is minimized by using an imaging geometry allowing intensity patterns defining the optical traps to be directly mapped into an addressable light shaping module. Hence, real time reconfigurable counterpropagating beam traps.

\section{EXTENDING FUNCITONALITY THROUGH THE SIDE VIEW OBJECTIVES}

Another important advantage of using low NA objectives is the increased workspace that gives more freedom on the sample containers or other contraptions for performing other biological studies. For example, advanced micro- 
spectroscopic or multi-photon characterization methods [6] can be implemented independently in the side-geometry. Cells can be subjected to stress and then characterized for different health indicators while trapped laser whose effect is known or being studied [7].

Recently we demonstrated dynamic axial stabilization of counter-propagating beam traps by tracking the trajectory of particles trough the side view imaging [8]. Additional stabilization is necessary as we stretch the axial trapping reach because regions with less intense light are less stable in holding particles in place. Computer vision tracks axial positions of multiple particles for use in a feedback algorithm that adjusts the respective counter-propagating beam pair intensities as needed (Fig 4). This allows particles to be moved-to or held in user defined axial positions within the $250 \mu \mathrm{m}$ height of a micro-fluidic channel.

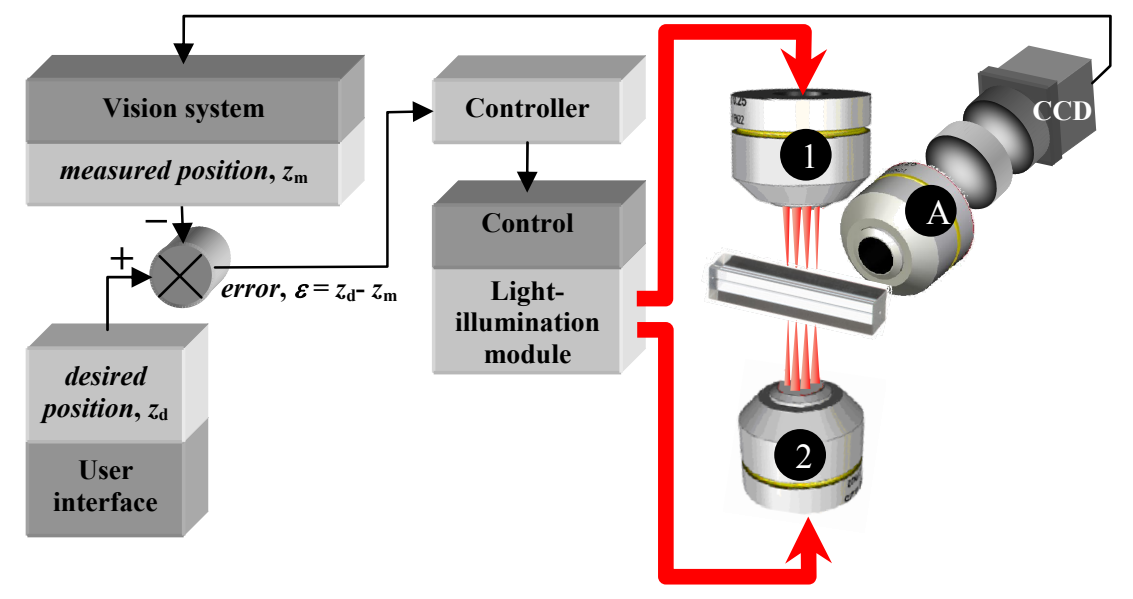

Figure 4. Schematic of the active trap stabilization on the BioPhotonics Workstation (BWS) [8]. An array of actively regulated traps are relayed through well-separated objectives ( 1 and 2$)$ that provides ample space for sideview microscopy (objective lens A, zoom lens and CCD camera). Computer vision provides real-time position feedback for regulating the traps.

\section{THE NEXT GENERATION BWS}

Up to this point, the BWS units in use are developed mostly with standard optical components and without requiring them to be compact or portable. The lack of such constraints allow high power optical traps, high quality imaging and an easily extensible hardware platform. User demands, however, are different and we may trade off some practical functionality that are not provided by a fully featured BioPhotonics Workstation. Developing a platform with a smaller footprint, for example, is a crucial step to make it more accessible for other intended users. This requires tweaking the BWS geometry and using more compact opto-mechanical components. Right now we are developing a miniature prototype BWS using standard and off-the-shelf components. 


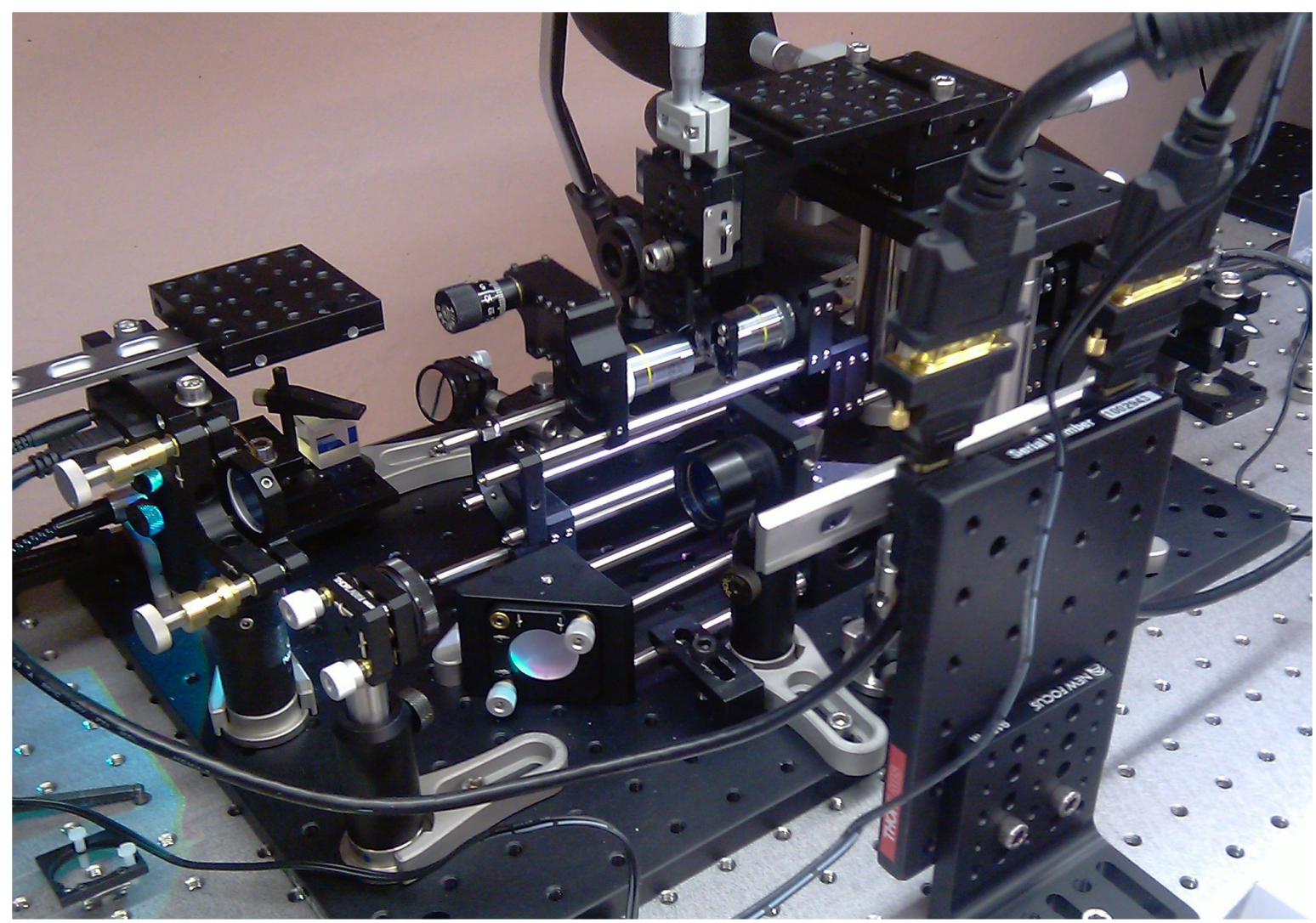

Figure 5. Miniaturised BWS. By using smaller opto-mechanical components, and tweaking the counterpropagating geometry, a smaller table-top BWS can be built.

In principle, opto-mechanical components can be made as small as desired using customized manufacturing techniques. The challenge in miniaturization lies with the laser sources since its output power is correlated with its size. Hence, an important step for miniaturization is to efficiently use the trapping lasers, allowing smaller power lasers to be used. The conventional CP geometry requires the trapped particle to be dislocated from the foci of the opposing objectives. Such a region however, has less intense light, and hence less trapping stiffness. One way of dealing with this without increasing the input laser power is to use a dynamic software feedback approach as mentioned in the previous section [8]. Another approach is to identify alternate regimes of stable trapping that exploit the higher intensities as the particle moves toward opposite image planes $[9,10]$. The results suggest that further optimizations for stability can be done modifying the 3D light distribution of the counter-propagating traps. 


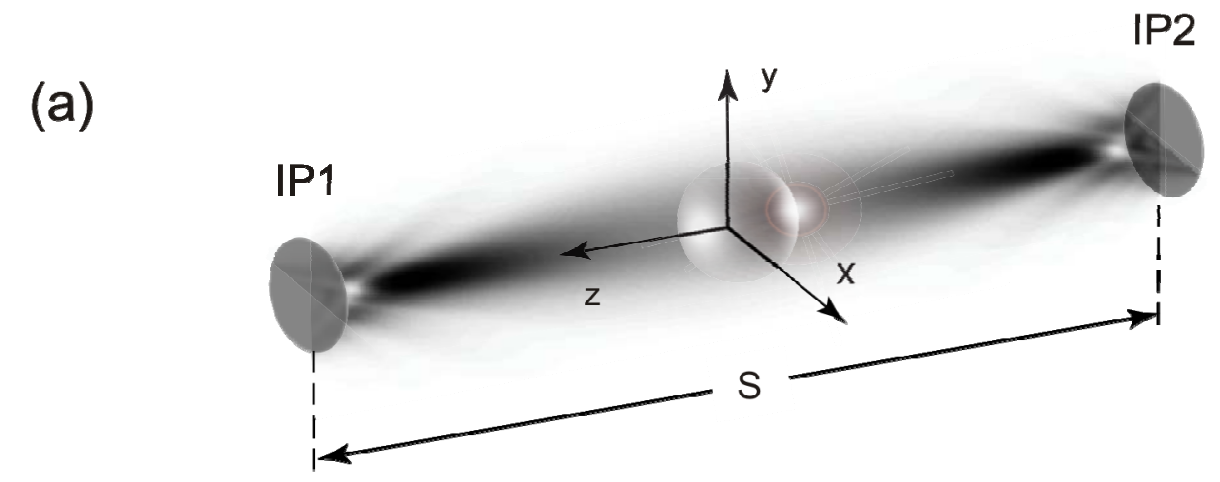

(b)

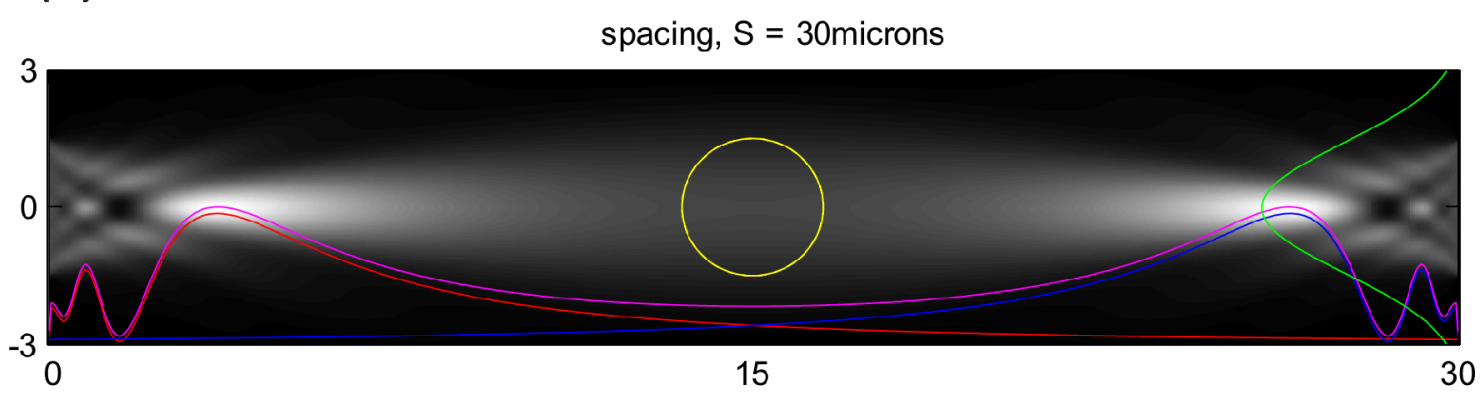

Figure 6. Analysis of the counter-propagating light distribution and forces reveals alternate regions for stable optical trapping near the image planes where light is more intense [9]. (a) 3D view of counter-propagating discshaped beams projected through opposite microscope objectives onto image planes, IP1 and IP2, separated by distance, s, to create a stable optical trap between the image planes. (b) Axial slice through the simulated volume intensity between two 3 micron diameter light discs; overlays show the expected stable trapping position for a microsphere, together with plots of intensity linescans (red: axial intensity of right-directed beam; blue: axial intensity of the left-directed beam; magenta: total axial intensity; green: transverse intensity linescan halfway between the discs)

Another area of improvement in terms of efficiency is with the input light that goes into the spatial light modulator. Laser output typically has a Gaussian amplitude distribution and a circular area while pixel oriented spatial light modulators are rectangular. Hence, losses are introduced when the input laser is magnified to illuminate the whole SLM surface. GPC can efficiently convert a Gaussian amplitude distribution into a rectangle that matches the SLM [11-13]

\section{SUMMARY}

The BioPhotonics Workstation enables long range 3D optical manipulation and extensible functionality through its side view imaging objectives. This is achieved by using a counter-propagating geometry coupled with long working distance optics. Direct mapping of the trap intensities to the sample allows real time manipulation of a plurality of independent traps, facilitating precise control and a rapid response in optical manipulation. Using extensions through the side view imaging, biological applications requiring different imaging requirements can be performed. Side view information has also been used to develop a stabilization scheme based on tracking the trapped particles' motions. Ongoing research aims at developing a Next Generation BioPhotonics Workstation that is more compact and efficient in using laser power. 


\section{References}

1. A. Ashkin, "Acceleration and trapping of particles by radiation pressure," Phys. Rev. Lett. 24, 4,156-159 (1970).

2. J. Glückstad, "Sorting particles with light", Nature Materials 3, 9-10 (2004).

3. P. Rodrigo, L. Gammelgaard, P. Bøggild, I. P.-Nielsen, and J. Glückstad, "Actuation of microfabricated tools using multiple GPC-based counterpropagating-beam traps," Opt. Express 13, 6899-6904 (2005).

4. P. Rodrigo, L. Kelemen, D. Palima, C. Alonzo, P. Ormos, and J. Glückstad, "Optical microassembly platform for constructing reconfigurable microenvironments for biomedical studies," Opt. Express 17, 6578-6583 (2009).

5. J. Glückstad, P. Rodrigo, I. P.-Nielsen, "Optical 3D manipulation and observation in real-time (Invited)", $J$. Robotics Mechatronics 18 (no.6), 692-697 (2006).

6. E. Papagiakoumou, F. Anselmi, A. Begue, V. de Sars, J. Glückstad, E. Isacoff, V. Emiliani, Nature Methods 7 , 848-854 (2010).

7. T. Aabo, I. P.-Nielsen, J. Dam, D. Palima, H. Siegumfeldt, J. Glückstad and N. Arneborg, "Effect of long- and short-term exposure to laser light at $1070 \mathrm{~nm}$ on growth of Saccharomyces Cerevisiae", J. Biomed. Opt. 15, 041505 (2010).

8. S. Tauro, A. Bañas, D. Palima and J. Glückstad, "Dynamic axial stabilization of counter-propagating beamtraps with feedback control," Opt. Express 18, 18217-18222 (2010).

9. D. Palima, T.B. Lindballe, M.V. Kristensen, S. Tauro, A. Banas, H. Stapelfeldt, S. R. Keiding, and J. Gluckstad, "Counter-propagating patterns in the BioPhotonics Workstation: getting more out of light for trapping and manipulation", Proc. SPIE 7762, $77620 \mathrm{U}$ (2010).

10. D. Palima, T.B. Lindballe, M.V. Kristensen, S. Tauro, H. Stapelfeldt, S.R. Keiding and J. Glückstad, "Alternative modes for optical trapping and manipulation using counter-propagating shaped beams," Journal of Optics (in press).

11. D. Palima and J. Glückstad, "Gaussian to uniform intensity shaper based on generalized phase contrast," Opt. Express 16, 1507-1516 (2008).

12. J. Glückstad and P. C. Mogensen, "Reconfigurable ternary-phase array illuminator based on the generalised phase contrast method", Opt. Comm. 173, 169-175 (2000).

13. J. Glückstad, "Adaptive array illumination and structures light generated by spatial zero-order self-phase modulation in a Kerr medium", Opt. Comm. 120, 194-203 (1995). 